\title{
I WATCH HER RIDE A CURRENT AROUND THE DELI
}

The body is fire and it's saying its language high and soundless.

It manifests as a force of nature, as if God could attach

a silencer to the wind, or teach a river to hush its warble over the rocks.

He sweeps a silencer over the barks and cries of birds,

gathers those cries cumulative, pours lightning cracks,

the rumblings of trains, the hiss from every cannonball heart.

He gathers them in a sack he lifts and pours into each of us

so we are rivers saying to each other, wind languages, the music of whales

across the table when we glance up from the steaming earth and-

hold onto it now lovejust look at each other. 